\title{
Resultados después de la implementación del test de DNA fetal libre circulante en sangre materna (DNA-Ic) para cribado de cromosomopatías fetales
}

\author{
Results after the implementation of the cell-free fetal DNA test in maternal plasma (cfDNA) in \\ screening for fetal aneuploidies
}

\author{
Eva María Soto-Sánchez', Carmen López-Gorosabel', Ana Belén Ibáñez-Santamaría', \\ Sandra Lagarejos- Bernardo', Juan José Hernández Aguado'
}

\begin{abstract}
Resumen
Objetivo: Evaluar la disminución de la tasa de técnicas invasivas de diagnóstico prenatal tras la introducción del cribado contingente de cromosomopatías con test de DNA fetal libre circulante (DNA-lc)y demostrar que este método de cribado es coste-efectivo. Método: estudio observacional prospectivo y estudio de coste efectividad. Primero se describen los resultados del cribado combinado en dos tiempos de primer trimestre desde febrero de 2008 a junio 2018 diez primeros años del hospital): 21744 cribados realizados de un total de 23000 partos. En abril de 2016 se implementa un modelo de cribado contingente de cromosomopatías con test de DNAlc (se oferta el test a pacientes con resultado de riesgo intermedio en el cribado combinado). En segundo lugar se analizan los resultados tras la implementación del test y se comparan dos períodos de tiempo con y sin cribado contingente (año 2015 con el período abril 2016 hasta marzo de 2019). Resultados: disminución total de las técnicas invasivas del 54\% por disminución de la tasa de amniocentesis, manteniéndose constante la tasa de biopsias coriales. La tasa de pérdidas fetales por técnica invasiva alcanza el 0\%. Usamos seis indicadores de calidad para evaluar el test. Se hanahorrado 70200 euros con la implementación del test de DNA-Ic. Discusión: el test de DNAlc resulta útil en el cribado contingente de cromosomopatías porque reduce la tasa de amniocentesis por indicación de alto riesgo y además es coste efectivo. El cribado combinado de primer trimestre es la técnica de elección para el cribado de aneuploidias. El test de DNAlc no puede sustituir al cribado combinado porque es caro, pero resulta muy útil si se realiza cribado contingente a la población seleccionada de riesgo intermedio.
\end{abstract}

Palabras clave: cribado; cromosomopatías; test de DNA fetal-Ic; TPNI; amniocentesis; biopsiacorial.

\begin{abstract}
Purpose: To describe the reduction in the rate of invasive techniques for prenatal diagnosis after the introduction of contingent aneuploidies screening with a circulating cell-free fetal DNA test (cfDNA) and demonstrate that this screening method is cost-effective. Method: a prospective observational study and a cost-effective study. First, we describe the results of combined screening in the first trimester of pregnancy, from February 2008 to June 2018: a total of 21,744 screenings from 23,000 childbirths. In April 2016, we implemented a model of contingent screening of aneuploidies with cfDNA tests (to patients with intermediate-risk results of the combined screening). Secondly, we describe the results after the implementation of the cfDNA test. We compare two periods of time, with and without contingent screening (the year 2015 with the period April 2016 to March 2019). Results: a reduction in invasive techniques of $54 \%$ due to the decrease in the rate of amniocentesis, keeping the number of chorial villosity biopsies constant. The fetal loss rate by invasive techniques has reached $0 \%$. We use six quality indicators to evaluate the test. We have saved 70,200 euros with the cfDNA test. Discussion: the cfDNA test is useful in the contingent screening of aneuploidies because it reduces the rate of amniocentesis as a result of the high-risk outcomes, and it is also cost-effective. The combined first trimester-screening is the method of choice for aneuploidy screening. The cDNA test cannot replace the combined screening because it is expensive, but it is useful in contingent screening when we select an intermediate-risk population.
\end{abstract}

Keywords: screening; aneuploidies; cf-DNA test; NIPT; amniocentesis; chorial villosity biopsy.

Fecha de envío: 24 de julio de 2019 - Fecha de aceptación: 19 de diciembre de 2019

(1) Servicio de Obstetricia y Ginecología. Hospital Universitario Infanta Leonor, Madrid, España

Autor de correspondencia: emaria.soto@salud.madrid.org 


\section{Introducción}

Las aneuploidías fetales más comunes son las trisomías de los pares 21, 18 y 13. La trisomía 21 o síndrome de Down ha sido uno de los objetivos prioritarios en la detección de aneuplodías por tratarse de la aneuploidía más frecuente en recién nacidos vivos. En la actualidad se observa un aumento de su prevalencia a causa, fundamentalmente, del incremento de la edad de las gestantes (aproximadamente 1,8\%) (Estrada et al., 2006).

Uno de los mayores avances en el cribado de aneuploidías es el test de ADN libre circulante (ADN-Ic) o test prenatal no invasivo (TPNI), disponible en el mercado desde 2011. Este test se basa en el conocimiento de que alrededor del 10\% del total de ADN-Ic en la sangre materna es de origen placentario, no necesariamente fetal. (Lo et al., 1997). Usando métodos de análisis de ADN es posible cuantificar la contribución fetal y estimar la probabilidad de que el feto esté afecto de un determinado defecto cromosómico.

Hay evidencia científica suficiente para afirmar que el test de ADN-Ic supone un cribado efectivo para las trisomías fetales más comunes (trisomías 21, 18 y 13) en gestaciones únicas y gemelares con una sensibilidad y especificidad del $99 \%$ para trisomía 21 y una tasa de falsos positivos menor a 0,1\% frente al 90 y $95 \%$ que presenta el cribado combinado de primer trimestre (CCPT) y la tasa de fasos positivos del 5\% para esta trisomía (Norton et al., 2012; Nicolaides et al., 2014; Gil et al., 2017).

Dado que la ecografía del primer trimestre aporta más información que el simple cribado de aneuploidías, la mayoría de sociedades científicas la consideran insustituible en el control gestacional (GAP SEGO, 2017; Salomón et al., 2017). De este modo, la estrategia más adecuada en el sistema público de salud, por coste efectividad, parece ser el modelo de cribado contingente con test de DNAIc. Según este modelo, se realiza el cribado combinado en dos tiempos (CCPT) a todas las gestantes y se ofrece el test de DNA-Ic en función de los resultados obtenidos con el cribado combinado a un grupo de gestantes seleccionadas según un punto de corte considerado de riesgo intermedio (Wright et al., 2015; Ashooret al., 2017; GAP SEGO, 2017).

En este artículo se analiza el cribado universal de la población con el CCPT durante los diez años de andadura del hospital desde su apertura en el 2008, y el impacto de la introducción del cribado contingente con test de DNA-Ic sobre las técnicas invasivas y coste efectividad en los últimos tres años.

\section{Materiales y Método}

En primer lugar se realiza estudio retrospectivo observacional lineal de 21744 gestantes a las que se realiza cribado combinado de primer trimestre (CCPT) sobre un total de 23000 partos desde Febrero de 2008 a Junio de 2018. El software utilizado de implementación del cribado es el Prisca, con centralización en laboratorio del Hospital Universitario Infanta Sofía de Madrid. Para realizar este CCPT se extrae analítica de sangre (beta-HCG y PAPP-A séricas) en la semana 10 y se realiza la ecografía del primer trimestre entre las semanas 11 y 13 semanas (CRL entre 45 y $86 \mathrm{~mm}$ ) acorde a los protocolos establecidos para tal determinación (GAP SEGO,2017; Nicolaides et al., 1992.). Se considera resultado positivo (alto riesgo) cuando el CCPT es mayor de $1 / 270$ y resultado negativo (bajo riesgo) cuando es menor de 1/270. El resultado alto riesgo es indicación de realización de técnica invasiva (amniocentesis o biopsia corial) para confirmación del diagnóstico y el resultado bajo riesgo permite el seguimiento habitual de la gestación (Stergiotou et al., 2016; Suela et al., 2017).

En segundo lugar, se realiza un estudio retrospectivo observacional lineal desde Abril de 2016 hasta Marzo de 2019 para evaluar el impacto de la introducción del cribado contingente con test de DNA-lc sobre las técnicas invasivas y sobre los costes económicos (estudio de coste-efectividad). En 2015 se realizó una propuesta a la gerencia del hospital para introducir el cribado contingente de cromosomopatías con test de DNA-lc. El Hospital Universitario Infanta Leonor es un hospital público con una población de referencia de 300000 personas, situado en el distrito de Villa de Vallecas de Madrid. El hospital aprobó la propuesta en 2016. Se estableció como único criterio para realización del TPNI un resultado intermedio en el CCPT definido como 1/100-1/270. Los resultados mayores de 1/100 se consideraron indicación de técnica invasiva, así como el hallazgo ecográfico en primer trimestre de anomalía ecográfica otranslucencianucal mayor de 3,5 mm. (Nicolaides et al., 2014). Los criterios fueron los mismos para gestaciones gemelares. El test aceptado fue NeobonaAdvanced $^{\circledR}$ (Synlab/lllumina), que utiliza una tecnología de secuenciación WGS paired-end y con capacidad de cribado de trisomías 21,18 y 13 y anomalías de los cromosomas sexuales.Este test tiene una sensibilidad global para la detección de trisomía 21 del $98,9 \%$ a un intervalo de confianza del $95 \%$, una especificidad $>99,9 \%$, una tasa de falsos positivos inferior al $2 \%$ y una tasa de falsos negativos inferior al 0,1\%, siempre que la fracción fetal (FF) detectada en sangre materna sea superior al $4 \%$. La sensibilidad a un intervalo de confianza del $95 \%$ es un poco menor para las trisomías 18 y 13 (90 y 95\%). La razón de probabilidad (likelihood ratio, LR) positivade que un feto esté afecto cuando un test de ADN-Ic da un resultado de alto riesgo es alrededor de 1.102, para trisomía 21, 740 para trisomía 18 y 700 para trisomía 13. (Nicolaides et al., 2014). 


\section{Resultados}

La figura 1 muestra la distribución de partos e IRCP (Priscas) realizados desde Febrero de 2008 a Junio de 2018. En total en este período se atendieron 23000 partos y se realizó un total de 21744 cribados, con una tasa media de 2330 cribados /año. El porcentaje total de población a la que se realizó el CCPT fue del 94,5\%. De la población cribada, se obtuvieron 802 casos de alto riesgo (3,69\% del total).

Desde la introducción del Test de DNA-Ic oTPNI se han realizado en total 157 TPNI en el período entre abril de 2016 y marzo de 2019, con una tasa media de 4,9 NIPT/mes. La fracción fetal media con la que se han emitido los informes del TPNI fue de 10,67 \%, siendo 3\% el mínimo requerido por esta técnica (Dondorpet al., 2015).

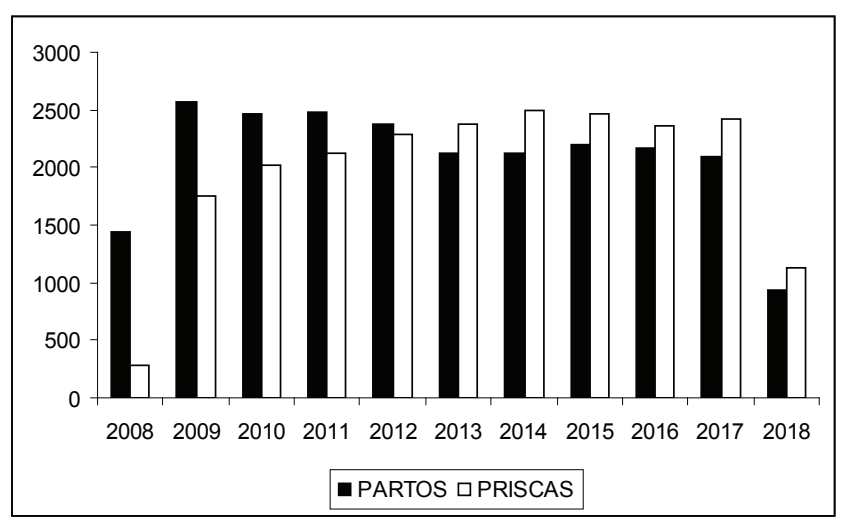

Figura 1: Distribución de partos y cribados combinados de primer trimestre (Priscas) en el Hospital Universitario Infanta Leonor desde febrero de 2008 a Junio de 2018.

Se obtuvieron 7 resultados de alto riesgo (NIPT positivo) lo que supone el 4,45\% de los realizados. En todos ellos se confirmó alteración cromosómica con cariotipo tras amniocentesis o biopsia corial, lo que supone una tasa de detección del 100\%.Todos los fetos con un resultado de bajo riesgo confirmaron al nacimiento la ausencia de cromosomopatías (tasa de falsos negativos del 0\%), tasa muy similar a la existente en la literatura internacional, inferior al 0,1\% (Dondorpet al., 2015, (Nicolaides et al.,2014) y una tasa de falsos positivos del $0 \%$, también similar a la publicada por otros autores, inferior al 2\% (Dondorpet al., 2015, (Nicolaides et al.,2014)

En la figura 2 puede verse la evolución de las técnicas invasivas en el hospital Infanta Leonor desde la introducción del cribado contingente de cromosomopatías con test de DNA-Ic, desde 126 técnicas en el año 2015, antes del cribado contingente a 72 en el año 2018 con el cribado contingente.

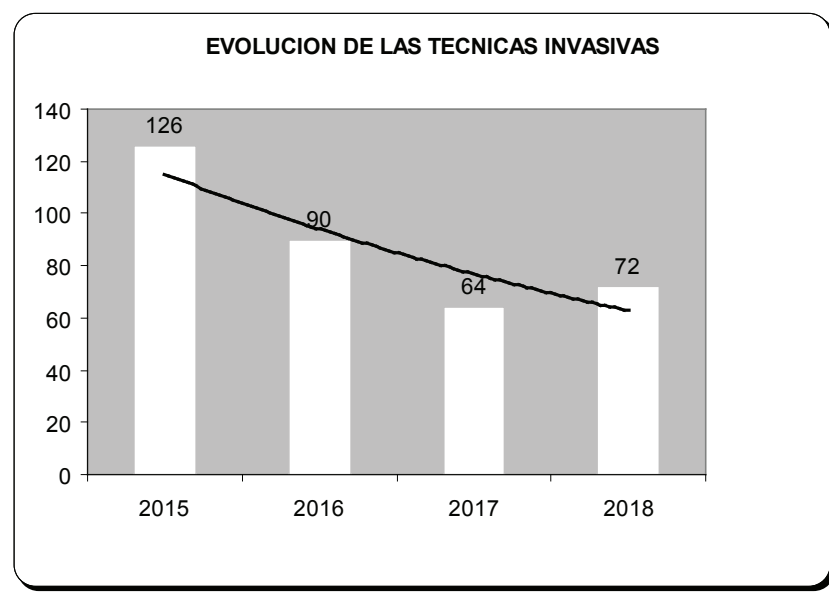

Figura 2: Evolución de las técnicas invasivas de diagnóstico prenatal en el Hospital Universitario Infanta Leonor tras la introducción del cribado contingente de cromosomopatías con TPNI.

En la figura 3 se analizan las diferencias encontradas entre las técnicas invasivas (amniocentesis y biopsias coriales) en los últimos años. Mientras que la tasa total de técnicas invasivas disminuye, especialmente por la reducción de la tasa de amniocentesis, la tendencia de las biopsias coriales aumenta significativamente. La reducción conseguida de las técnicas invasivas ha sido del $31 \%$ el primer año, $54 \%$ el segundo y $51 \%$ el tercer año.

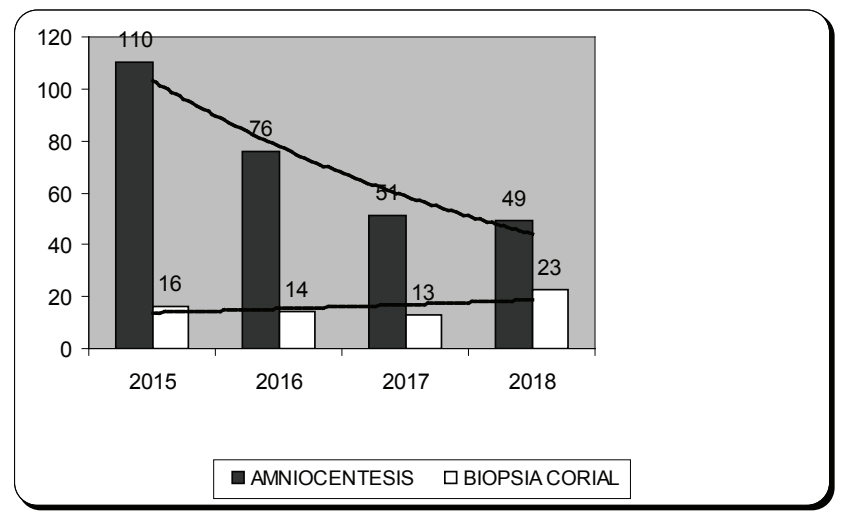

Figura 3: Diferencias en la evolución del número de amniocentesis y biopsias coriales en el Hospital Universitario Infanta Leonor tras la introducción del cribado contingente de cromosomopatías con TPNI.

La tasa global de pérdidas fetales por técnicas de diagnóstico prenatal invasivas en nuestro centro hasta el año 2016 se situaba en $0,7 \%$. La tasa actual, tras la introducción del cribado contingente, se sitúa en $0 \%$. 
Se han utilizado seis indicadores de evaluación del TPNI:

1-Porcentaje de gestantes que cumplen criterios para ofrecer estudio de ADN-Ic en sangre materna (los que se han definido previamente como riesgo intermedio) sobre el total de gestantes con riesgo alto: $57,2 \%$.

2-Porcentaje de gestantes que cumplen criterios para ofrecer estudio de ADN-Ic en sangre materna y aceptan la realización del estudio: $100 \%$.

3-Porcentaje de test de DNA-Ic en sangre materna "sin resultado" en un primer test (tasa de repeticiones): 0,79\%.

4-Porcentaje de test de DNA-Ic en sangre materna positivo (riesgo alto): $4,45 \%$.

5-Porcentaje de gestantes con test de DNA-Ic en sangre materna positivo (riesgo alto) que acepta realizarse la amniocentesis: $100 \%$.

6- Porcentaje de gestantes con test de DNA-lc en sangre materna positivo (riesgo alto) y amniocentesis en las que se confirma la anomalía cromosómica: $100 \%$.

Finalmente, en la figura 4 puede verse el impacto económico que la introducción del cribado contingente con TPNI ha tendido en nuestro hospital. Se ha situado el coste del test en 300 euros y de la técnica invasiva en 900 euros. De este modo, si añadimos los casos de TPNI positivos, a los que tuvimos que hacer las dos técnicas, al total del coste de los TPNI realizados, y tenemos en cuenta las amniocentesis que han dejado de realizarse por un resultado de TPNI bajo riesgo, el total supone un ahorro de 70200 euros en tres años.

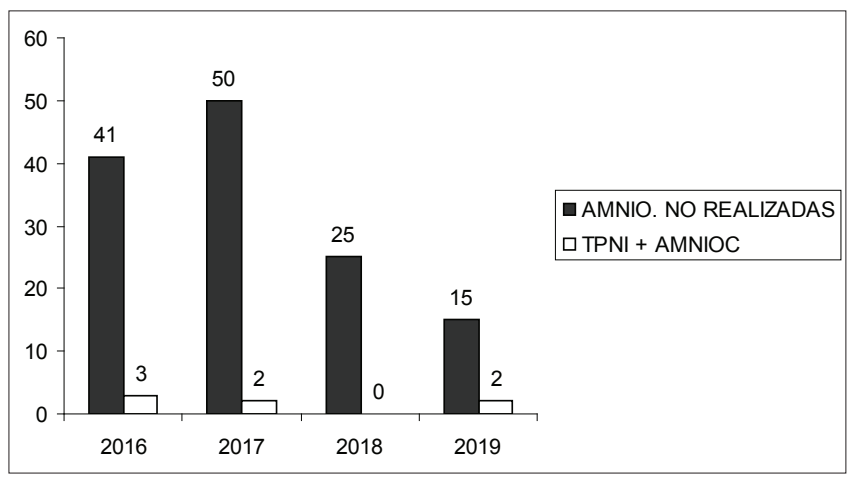

Figura 4: Estudio de coste efectividad: volumen de amniocentesis no realizadas (amnio. no realizadas) tras introducción del TPNI y número de TPNI postivos a los que se realizó ambas técnicas (TPNI + amnioc).

\section{Discusión}

En nuestro centro, la introducción del cribado contingente de cromosomopatías con TPNI ha supuesto una reducción considerable en la tasa de amniocentesis.Así mismo, se ha producido una reducción significativa en la tasa de pérdidas fetales (de 0,7\% a 0\%).

Los indicadores de evaluación nos permiten considerar el test de DNA-lc como una prueba muy útil en el cribado de aneuploidías. Permite adelantar el diagnóstico de las mismas y reducesignificativamente la tasa de amniocentesis por indicación de cribado combinado de alto riesgo.

Este test presenta además una excelente aceptabilidad, tasa de detección, sensibilidad y especificidad y muy bajas tasas de falsos positivos y falsos negativos.

La tasa de repetición en nuestro centro ha resultado muy baja (sólo un caso de 157 que fue debido a una gestación gemelar no conocida previamente con un gemelo evanescente y en la que la determinación del sexo fetal no fue posible).Aunque esta tasa ha sido muy baja en nuestro estudio, hasta en un $4 \%$ de los casos los resultados pueden no ser concluyentes si la cantidad de DNA-Ic es escasa. En un 65\% de los casos se obtendrán resultados satisfactorios en un segundo análisis con una nueva extracción sanguínea materna. (Stergiotouet al., 2016; Suela et al., 2017). Otra posibilidad de limitación del test debida a escasa fracción fetal puede producirse en caso de obesidad severa materna (índice de masa corporal, IMC, superior a 40).

Existen otras limitaciones asociadas a la técnica como imposibilidad de obtener resultado si se ha realizado en los tres meses previos una transfusión de sangre, trasplante, terapia celular, inmunoterapia o radioterapia.

El ADN-Ic que se analiza es de origen placentario y, por tanto, en algunos casos los resultados obtenidos pueden no coincidir con el complemento genético del feto. Esto ocurre con una incidencia inferior al $2 \%$ de los casos. Es por tanto importante informar siempre de que se trata de una test de cribado con una alta sensibilidad y especificidad pero en ningún momento es un test diagnóstico y ante la sospecha de anomalía posible, debe siempre confirmarse con una técnica invasiva (amniocentesis o biopsia corial). Por tanto, el test de DNA no detecta mosaicos ni trisomías parciales, es decir, que cuando las células no son todas iguales, y existen células con cromosomas anormales, habitualmente no puede detectarse por este método. No provee información sobre otras anomalías de los cromosomas, en general, mucho más raras. Tampoco provee 
información sobre defectos físicos o del crecimiento. Por lo tanto, sigue siendo recomendable realizar las ecografías protocolizadas del embarazo según criterios de la sociedad científica correspondiente.

Desde el punto de vista económico, el cribado contingente con test de DNA-Ic permite ahorrar, y lo que es más importante, la reducción de las tasa de amniocentesis, permiten también disminuir el número de perdidas fetales debidas a la técnica.

Aunque hoy en día el método de elección para el cribado global de cromosomopatías sigue siendo el cribado combinado por la alta tasa de detección y el bajo coste, el cribado contingente con test de DNA asociado permite disminuir costes y mejora la tasa de detección. Por lo que es posible que en un futuro cercano, si se consigue abaratar aún más este test, pueda introducirse como cribado global junto con la ecografía.

Recientemente hemos asistido a la introducción oficial del cribado contingente de cromosomopatías con TPNI en la Comunidad de Madrid, con nuevos criterios de inclusión, lo que seguramente nos permitirá reducir aún más la tasa de amniocentesis en nuestro centro.

\section{Conclusión}

El cribado contingente con test de DNA-Ic permite ahorrar, reducir latasa de amniocentesis y el número de pérdidas fetales debidas a la técnica.Se trata, no obstante, de un test de cribado con alta tasa de detección, pero siempre necesita confirmación diagnóstica con técnica invasiva ante la sospecha de anomalía cromosómica.

Con la introducción oficial del cribado contingente de cromosomopatías con TPNI en la Comunidad de Madrid, con nuevos criterios de inclusión, se podrá reducir aún más la tasa de amniocentesis.

\section{Contribuciones y conflictos declarados por los autores}

Todos los autores han participado en la realización del cribado contingente.

La fuente de financiamiento es el Sistema Público de Salud de la Comunidad Autónoma de Madrid.

No hay conflictos de interés declarados por los autores.

\section{Referencias}

Ashoor G, Syngelaki A, Wagner M, Birdir C \&Nicolaides KH. (2012). Chromosome-selective sequencing of maternal plasma cell-free DNA for first trimester detection of trisomy 21 and trisomy 18. Am J ObstetGynecol 206, 322-5.
Dondorp W, de Wert G, Bombard Y, Bianchi DW, Bergmann C et al. (2015). European Society of Human Genetics; American Society of Human Genetics. Non-invasive prenatal testing for aneuploidy and beyond: challenges of responsible innovation in prenatal screening. Eur J Hum Genet 23, 1438-1450.

Estrada M D, Guillén M, Iruretagoiena M L, Taboada J, López de Argumedo M; Lapuente J, Gutiérrez M, Feijas A, Cuntín L, Gestoso J, García A \&Cerdá T. (2006). Descripción del estado de situación del cribado prenatal de las cromosomopatías fetales más frecuentes -principalmente Síndrome de Down- en el Estado español y propuestas de mejora en la práctica clínica habitual. Madrid: Plan de Calidad para el Sistema Nacional de Salud. Ministerio de Sanidad y Consumo. Informes de Evaluación de Tecnologías Sanitarias,AATRM núm. 2006/03.

Gil MM, Accurti V, Santacruz B, Plana MN \& Nicolaides KH. (2017). Analysis of Cell-Free DNA in maternal blood in screening for aneuploidies: Updated Meta-Analysis. Ultrasound ObstetGynecol 50, $302-314$.

Grupo expertos SESEGO y SEMEPE consensuado con AEDP. (2017). Guía de asistencia práctica: Cribado y Diagnóstico precoz de anomalías genéticas.Sociedad Española de Ginecología y Obstetricia $61,605-629$

Lo YM, Corbetta N, Chamberlain PF, Rai V, Sargent IL, Redman CW \&Wainscoat JS. (1997). Presence of fetal DNA in maternal plasma and serum Lancet 350, 485-7.

Nicolaides KH, Azar G, Byrne D, Mansur C \& Marks K.(1992). Fetal nuchaltranslucency: ultrasoundscreeningforchromosomaldefects in firsttrimester of pregnancy. BMJ 304, 867-9.

Nicolaides KH, Syngelaki A, Poon LC, Gil MM \& Wright D.(2014). First-trimester contingent screening for trisomies 21, 18 and 13 by biomarkers and maternal blood cell-free DNA testing. FetalDiagnTher 35,185-92.

Norton ME, Brar H, Weiss J, Karimi A, et al. (2012). Non-Invasive Chromosomal Evaluation (NICE) Study: results of a multicenter prospective cohort study for detection of fetal trisomy 21 and trisomy 18. Am J ObstetGynecol 207137.e1-8.

Salomón LJ, Alfirevic Z, Audibert F, Kagan KO, Paladín D, Yeo G, Raine-Fenning N. \&ISUOG Clinical Standards Committee. (2017). ISUOG Consensus Statement on the impact of non-invasive prenatal testing (NIPT) on prenatal ultrasound practical. Ultrasound ObstetGynecol 49, 815-6. 
Soto et al.

Stergiotou I, Borobio V, Bennasar M, Goncé A, Mula R, Nuruddin M, soler A \& Borrell A. (2016). Transcervical chorionic villus sampling: a practical guide. J Matern Fetal Neonatal Med 29, 1244-51.

Suela J, López-Expósito I, Querejeta ME, Martorell R, Cuatrecasas E, Armengol L, Antolín E, Domínguez Garrido E, Trujillo-Tiebas MJ,
Rosell J, García Planells J. \& Cigudosa JC (2017). Grupo de diagnóstico prenatal del INGEMM; Grupo de genética prenatal del Hospital Clínico San Carlos. Med Clin 148, 328.e1-328.e8.

Wright D, Wright A \&Nicolaides KH. (2015). A unified approach to risk assessment for fetal aneuploidies. Ultrasound ObstetGynecol 45, 48-54. 